\title{
La administración de proyectos de infraestructura en el Ministerio de Educación Pública de Costa Rica
}

\author{
Infrastructure project management at the Ministry \\ of Public Education of Costa Rica (MEP)
}

\author{
Maikol Víquez-Duarte \\ Académico, Escuela de Ciencias de la Administración \\ Universidad Estatal a Distancia \\ San José, Costa Rica \\ mviquezd@uned.ac.cr \\ Víctor Orozco-Delgado \\ Académico, Escuela de Administración Educativa \\ Universidad de Costa Rica \\ San José, Costa Rica \\ victor.orozco@ucr.ac.cr
}

Recibido - Received: 21 / 02 / $2020 \quad$ Corregido - Revised: 20 / 05 / 2020 Aceptado - Accepted: 01 / 06 / 2020

DOI: https://doi.org/10.22458/ie.v22i32.2819

URL: https://revistas.uned.ac.cr/index.php/innovaciones

\begin{abstract}
Resumen: Mediante una reconstrucción histórica con consideraciones jurídicas inherentes a la realidad de los responsables de ejecutar la administración pública costarricense, se busca describir de forma práctica la realidad de la organización del personal administrativo de una entidad pública, la cual cuenta con una de las más grandes planillas a nivel centroamericano y se enfrenta al desafío de atender la que parece una interminable gestión: desarrollar infraestructura educativa en el país. Hecha la revisión documental correspondiente y el análisis de la misma, se evidencia una clara intención del Ministerio de Educación Pública de buscar alternativas distintas para la atención de la problemática relacionada con la construcción y mantenimiento de obra; sin embargo, la problemática continúa vigente, pese a que, a partir del año 2012, se acudió a instrumentos alternativos para la construcción de edificios escolares, como es la constitución de un fideicomiso para el desarrollo de infraestructura educativa, el cual es un importante esfuerzo ministerial, pero como primera experiencia y al estar en cumplimiento de su periodo de ejecución, los resultados aún no han sido los esperados, básicamente por el plazo de entrega de obras y por el incremento en los costos inicialmente proyectados.
\end{abstract}

Palabras clave: edificio escolar, eficiencia de la educación, organismo educacional gubernamental, organización gubernamental, proyecto de desarrollo

Summary: Through a historical reconstruction with legal considerations inherent to the reality of those responsible for executing the Costa Rican public administration, the aim is to describe in a practical way the reality of the organization of the administrative staff of a public entity, which has one of the largest payrolls at the Central American level and faces the challenge of attending to what seems like endless management: developing educational infrastructure in the country. After the corresponding documentary review and analysis, a clear intention of the MEP to search for different alternatives to deal with the problems related to the construction and maintenance of the work is evident. However, the problem continues, despite the fact that, since 2012, alternative instruments were used for the construction of school buildings, such as the constitution of a trust for the development of educational infrastructure, which is an important ministerial effort. However, as a first experience and being in compliance with its execution period, the results have not yet been as expected, basically due to the deadline for the delivery of works and the increase in costs initially projected.

Key Words: school building, education efficiency, government educational agency, government organization, development project 


\section{INTRODUCCIÓN}

La eficacia supone que la organización y función administrativa deben estar diseñadas y concebidas para garantizar la obtención de los objetivos, fines y metas propuestos y asignados por el propio ordenamiento jurídico. En este sentido, la Constitución Política en su artículo 11, establece: "La Administración Publica en sentido amplio, será sometida a un procedimiento de evaluación de resultados y rendición de cuentas" (Arce, 2004, p. 3, párrafo segundo de la Constitución Política).

Las obras de infraestructura pública son una necesidad entendida por toda la población, que recurre de manera democrática a escoger cada cuatro años a quienes consideran más capaces para poder concretarlas. Así, los gobernantes se enfrentan al requerimiento fundamental de contar con el recurso humano competente para poder formular, ejecutar y administrar las obras durante su vida útil. Sin embargo, una obra de infraestructura se reviste de una gran singularidad, ya que para cada obra se requiere llevar a cabo tareas como: selección de la ubicación idónea, elaboración de anteproyectos y planos, labores de obtención de permisos, presupuestación, comunicación con los involucrados, procedimientos legales, contrataciones, construcciones, inspecciones y finalmente la administración especializada y el mantenimiento de los edificios escolares.

Los funcionarios públicos en quienes recae la responsabilidad de ejecutar todas estas tareas se encuentran inmersos dentro de la organización gubernamental, típicamente encabezada por una administración que inicia generalmente con una distribución de los poderes y que identifica sectores que considera estratégicos para poder cumplir con su contrato social asignándoles una rectoría.

A partir de este grado de libertad frente a los órganos centrales, los encargados de las instituciones recurren a sus leyes constitutivas, reglamentos, manuales, así como cualquier otra legislación vinculante con su actividad principal. La celeridad en la ejecución de estas obras depende en gran medida de la forma en que se organice la administración pública.

En Costa Rica, El Ministerio de Educación Pública es el principal organismo educacional gubernamental responsable de atender la necesidad de educar a la población. Sin embargo, como institución pública debe velar por el cumplimiento de las obligaciones jurídicas, que de alguna forma impactan en los resultados esperados; por lo tanto, aquellos que atienden la tarea de gerenciar la administración pública en un entorno cambiante, tratan de ajustar estos elementos con la intención de alcanzar los objetivos propuestos, como puede ser el desarrollo de infraestructura educativa.

La memoria institucional del Ministerio de Educación Pública de Costa Rica 2006-2010, inicia dando cuenta de las reformas institucionales para una mejor gestión administrativa, señalando lo siguiente:

Un diagnóstico realizado como base para la estrategia del Sector Educación 2006-2010 identificó una serie de rigideces estructurales que venían debilitando, de manera sistemática, la capacidad de gestión, tanto en el ámbito académico como administrativo. Una de esas limitaciones, quizás la más evidente para los usuarios -internos y externos- estaba relacionada con un estilo de administración poco sensible ante las necesidades de las comunidades educativas que se reflejaba en una organización centralista, vertical, fragmentada y dispersa; en donde muchos de los problemas de gestión estaban asociados a una limitada capacidad de planificación, coordinación, ausencia de procedimientos y mecanismos de control, así como por el hecho de que el grueso de los trámites se realizaban de forma manual. (MEP, 2010, p. 17)

El presente trabajo tiene por objetivo dilucidar la relevancia de la organización del Ministerio de Educación Pública de Costa Rica en la atención de proyectos de desarrollo de infraestructura educativa, como un medio que permita mejorar la eficiencia escolar en el país. 


\section{Método}

Se presenta una reconstrucción histórica de la forma en que la administración del Ministerio de Educación Pública del gobierno de Costa Rica ha buscado suficientes y adecuadas instalaciones escolares para brindar el servicio educativo. Se da seguimiento a la institución que actualmente existe para resolver el desafío desde su creación en 1965 Ley N 3481 hasta su última versión legalmente constituida.

Se trata de estudiar un tema pasado de envergadura, pero su análisis se hace a efectos de intentar entender nuestra realidad presente, proyectándonos al futuro. Es decir, un trabajo histórico alcanza interés cuando nos sirve para entender por qué estamos cómo estamos o por qué hemos llegado a donde hemos llegado, motivo por el cual una denominación más accesible es la de "estudio evolutivo". (Tantaleán, 2016, p. 27)

Como textos fundamentales para el trabajo se consideran los decretos organizacionales del Ministerio de Educación Pública de Costa Rica, complementados con las reflexiones y críticas que emanan de las memorias o informes de labores confeccionados por las diferentes administraciones a cargo de la institución.

El estudio trata sobre la administración entendida como la capacidad para adoptar decisiones o acciones necesarias y realizarlas (Jinesta, 2011), en este caso concreto enfocándonos en la administración de proyectos de infraestructura educativa.

Bajo esta premisa, se analizaron dos tipos de documentos: los decretos publicados en el diario oficial La Gaceta que afectaron la organización del MEP desde su creación en 1965 a la actualidad y que señalan las obligaciones normadas para la administración de proyectos de infraestructura educativa; y las memorias institucionales existentes en el mismo periodo de tiempo y en las cuales las administraciones del MEP expusieron y justificaron los resultados conseguidos durante diferentes gobiernos.

\section{LITERATURA SOBRE EL TEMA}

El establecimiento de la educación como prioridad nacional se manifestó mediante la abolición del ejército y la inclusión de un capítulo de educación y cultura en la constitución política de 1949.

Esta normativa le otorga relevancia constitucional a un Consejo Superior de Educación con la responsabilidad de dirigir la enseñanza oficial. El órgano, creado en 1951 mediante Ley $N^{\circ} 1362$ y presidido por el Ministro de Educación, debe conocer, entre otros temas, sobre los planes de edificación escolar, los proyectos para la creación de nuevos tipos de escuelas y colegios, los planes para incrementar la educación pública, los proyectos de ley, reglamentos, planes de estudio y programas a que deben someterse los establecimientos educacionales y resolver sobre los problemas de correlación e integración del sistema educacional (Ley Creación del Consejo Superior de Educación Pública, N¹362, 1951).

Además, la Constitución organizó la educación pública como un proceso integral correlacionado en sus diversos ciclos, desde preescolar hasta universidad; con el fin de fortalecer el criterio, en el año de 1957, los legisladores decretaron la Ley Fundamental de Educación N²160, con la cual se gradúa la educación escolar conforme con el desarrollo psicobiológico de los educandos y comprendiendo los siguientes niveles: educación pre-escolar, educación primaria, educación media y educación superior.

En el capítulo VIII de la Ley Fundamental de Educación, se señala que cada institución de enseñanza contará con una junta administrativa o junta de educación nombrada por la municipalidad. Se indica que las juntas serán dotadas con rentas provenientes del presupuesto nacional, de las municipalidades, de las instituciones autónomas y de otras fuentes de carácter especial, contando también las juntas con 
capacidad para adquirir derechos y contraer obligaciones y que, en cuanto a bienes inmuebles se refiere, solo pueden adquirir aquellos que sean necesarios para el cumplimiento de sus fines (Ley Fundamental de Educación, N²160, 1957).

En 1965 el gobierno de Costa Rica constituye un órgano técnico para administrar el sistema educativo, modernizar las oficinas existentes y proyectarse fuera del Valle Central. El mecanismo utilizado para crear la entidad, fue la Ley N3 3481 denominada Ley Orgánica del Ministerio de Educación Pública. Esta regulación establecía, como función del titular superior del ramo, servir de vínculo entre el Poder Ejecutivo y las demás instituciones que trabajaban en el campo educativo y cultural, tanto públicas como privadas, nacionales e internacionales. El resto de actividades fueron delegadas en asesorías técnicas y organismos administrativos.

A las asesorías técnicas para cada nivel de enseñanza les correspondía la administración de los planes y programas educativos, relacionados con los distintos niveles y las diversas ramas del sistema escolar. Además, se les otorgó el carácter de tales a las comisiones que se crearon para el estudio de los problemas específicos de la educación.

Por organismos administrativos, en esta ley se determinaron los siguientes: la Oficialía Mayor, el Departamento de Administración General de la Enseñanza, el Departamento de Formación Profesional Docente, el Departamento de Personal, el Departamento Financiero y el Departamento de Extensión Cultural y Bibliotecas. A cada uno de estos órganos, se les establecieron responsabilidades en esta misma ley, para las cuales se les otorgó la posibilidad de crear secciones, mediante los respectivos reglamentos.

Con subordinación jerárquica al Ministro, cada organismo tendría un director y cada sección un jefe, con su correspondiente personal. La estructura, las funciones y cualquier otro aspecto a lo interno de cada instancia, relacionados con la marcha general del Ministerio se regirían por los reglamentos que se dictaren en cada caso.

Así entendido, y al amparo del artículo $N^{\circ} 140$ de la Constitución que en su inciso $N^{\circ} 18$ señala que es atribución o deber del presidente y respectivo ministro: “Darse el Reglamento que convenga para el régimen interior de sus despachos y expedir los demás reglamentos y ordenanzas necesarias para la pronta ejecución de las leyes"; significó el inicio del proceso organizativo de la estructura ministerial, que se intensificó con el crecimiento y evolución de la población y en consecuencia de la administración pública costarricense.

En 1971, se crea el Ministerio de Cultura, trasladándose la materia que competía al Departamento de Extensión Cultural y Bibliotecas a esta instancia. También en ese año con la Ley № 3155 denominada: Crea el Ministerio de Transportes en sustitución del actual Ministerio de Obras Públicas, se establecen elementos propios de la forma básica de desarrollar obras públicas para brindar el servicio educativo y se establece que para los efectos de la presente ley, se considerará que las edificaciones para la enseñanza primaria, media o vocacional comprenden la planta física, las obras complementarias y los bienes muebles accesorios que la complementan. Además se indica que ninguna persona o entidad pública o privada podrá construir edificaciones de esta naturaleza sin la previa aprobación del Ministerio de Obras Públicas y Transportes.

En lo referente al financiamiento se autorizó al Ministerio de Obras Públicas y Transportes (MOPT) para destinar materiales y servicios obtenidos a través de las partidas consignadas en los presupuestos ordinarios y extraordinarios, a la ejecución de obras o actividades de bien público que pudiera realizar conjuntamente con municipalidades, juntas de protección social, juntas de educación, juntas administrativas de colegios dedicados a la enseñanza o de instituciones públicas de desarrollo comunal.

También señala la ley que el MOPT puede entregar los materiales y servicios a las citadas instituciones como colaboración en la ejecución de las obras; y contratar directamente con ellas la construcción, 
mantenimiento y mejoras de edificios públicos, para toda clase de obras públicas, así como la fabricación de mobiliario, cubriendo los gastos de los contratos por medio de las partidas destinadas a obras por licitación.

Finalmente, en septiembre de 1971, se crea mediante decreto № 2001-T la Dirección General de Edificaciones Nacionales, señalándosele la obligación de construir, mejorar y mantener las edificaciones y demás obras públicas no sujetas a disposiciones legales especiales y de vigilar que se les dé el uso adecuado y se agrega que la planificación de estas obras se hará conjuntamente con los organismos a los cuales incumbe su funcionamiento, operación y administración.

Para 1972, el Ministro de Educación Pública, señor Uladislao Gámez Solano, contaba con una estructura organizacional de la administración central (figura 1) instituida según el cambio de estructura del sistema educativo y considerando la división en los dos grandes sectores de asesoría técnica y órganos administrativos.

Figura 1. Organigrama Ministerio de Educación Pública, año 1973

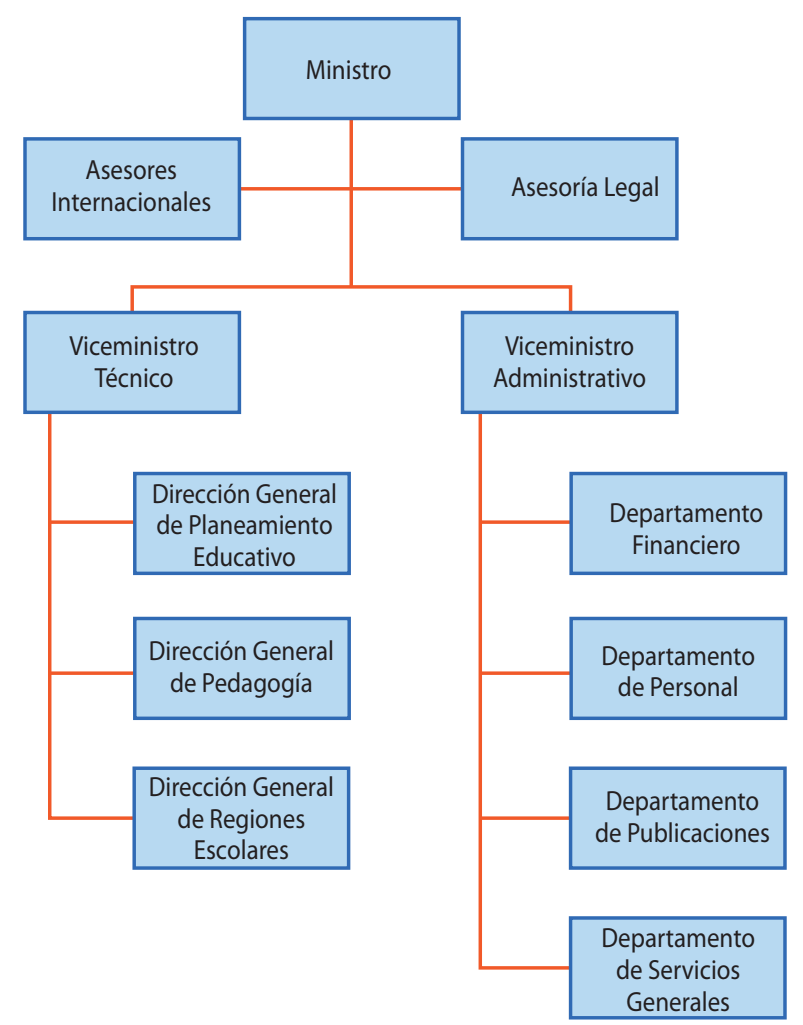

Fuente: Elaboración propia a partir de la propuesta de Gámez (1981).

Para el Ministro, la organización se llevaba a cabo con base en las siguientes cinco funciones fundamentales, que eran identificadas cada una, con un correspondiente sector funcional, a saber:

1. Órganos de decisión: el ministro y dos viceministros uno por cada sector, un viceministro del sector técnico-pedagógico y un viceministro de lo propiamente administrativo. De acuerdo con don Uladislao se llegó a esta agrupación por sectores al cabo de un profundo estudio de reforma administrativa en 1972, que consideró las variadas funciones del ministro y la necesidad de delegar funciones político-administrativas. 
2. Órganos de planificación: integrado por unidades destinadas a preparar y fundamentar las decisiones, programar su ejecución y evaluar su realización como base para nuevas decisiones a nivel de la conducción del sistema educativo nacional. A cargo de la planificación se estableció la Dirección General de Planeamiento, conformada por las siguientes unidades: unidad de investigaciones pedagógicas, unidad de documentación pedagógica, unidad de estadística y estudios de expansión, unidad de planeamiento físico, unidad de administración, unidad de financiamiento y costos, unidad de contenidos y métodos, unidad de supervisión y organización y la unidad de recursos humanos.

3. Órganos de asesoría: responsables de orientar a los educadores hacia la consecución de los objetivos de la educación, estaban dirigidos por la Dirección General de Pedagogía dividida en los departamentos de: Asesoría Técnica-docente, TV Educativa, Departamento de Orientación, Educación Especial, Educación de Adultos y Educación Superior.

4. Órganos de ejecución y supervisión: el conjunto de órganos responsable de poner en ejecución los planes y programas de acción correspondiente a la política educativa aprobada, así como de controlar el cumplimiento de disposiciones legales, reglamentarias y las emanadas de la autoridad competente del Ministerio. Presidiendo estos órganos se encuentra la Dirección General de Regiones Escolares que coordina a los departamentos de: Educación General Básica, Educación Académica y Educación Técnica.

5. Órganos de apoyo: dirigidos y coordinados por el viceministro administrativo, tienen la función fundamental de suministrar y mantener servicios, bienes y recursos o llevar a cabo cualquier tarea necesaria para la prestación del servicio educativo. Integran este sector los departamentos de: Personal, Publicaciones, Servicios Generales y Financiero. (Gámez, 1981, p. 156)

Entre 1970 y 1974, Uladislao Gámez Solano ejercía su tercer periodo como ministro del ramo, durante su administración se escribió el documento denominado: Plan Nacional de Desarrollo Educativo, en el que se refiere a la reestructuración administrativa e indica que la ejecución de un plan de desarrollo educativo requiere una transformación correlativa del sistema administrativo.

Este documento, al que en 1973 se le procuró el grado de decreto $N^{\circ} 3333$, señala que no existían en esa época objetivos explícitos de la administración educativa y que esta funcionaba simplemente para mantener el sistema escolar, sirviéndose de textos legales y de precedentes, explica también el texto que los órganos más modernos y dinámicos, como la Oficina de Planeamiento y el Departamento de Curriculum, surgieron en los últimos años, y al ser introducidos en un cuerpo antiguo sin modificarlo en lo esencial, provocan lógicamente duplicaciones y fricciones que solo se resolverían correctamente por medio de una reestructuración (Decreto Nº3333-E, 1973).

El decreto manifiesta la necesidad, detectada en el periodo, de asignar correctamente sus funciones al personal correspondiente a través de una definición de atribuciones y que debe formarse un cuerpo administrativo vigoroso, compuesto de los mejores profesionales y técnicos en sus respectivos campos. Señala entonces que debe contarse con un esquema claro de la organización y con un manual de actuación para cada órgano y cada puesto. Indica, finalmente, como transcribiendo la visión del exministro, lo siguiente:

En el trazado de un programa de reestructuración administrativa se ha preferido proceder de abajo arriba, es decir, del nivel local al regional y de éste al nacional. Ello responde al objeto básico de la administración que desea: las instancias superiores de la administración se organizarán según las necesidades de servicios que presenten las inferiores y no según conceptos apriorísticos de "jerarquía" propios de una organización autoritaria. En educación el trabajo real se hace en cada aula, no en el Ministerio de Educación; es éste el que debe ponerse al servicio de las necesidades de aquéIlas. (Decreto N 3333-E, 1973) 
De acuerdo con el exministro Gámez, la Administración 1974-1978 se apartó del organigrama (figura 2), se observa el reemplazo del espacio de Consejo de los Asesores Internacionales y la ubicación en esa posición de la Dirección General de Planeamiento Educativo, pasando a ser dependencia directa del ministro, al igual que una unidad de asesorías y comisiones ministeriales sin especificar. Además, se aprecia entre los principales cambios que el viceministerio técnico cuenta con tres direcciones: Dirección General de Educación Técnica, Dirección General de Educación Académica y la Dirección General de Acción y Servicios Docentes. Por otro lado, al viceministerio administrativo antes descrito se le incorpora el Departamento de Suministros Escolares y la asesoría legal.

Figura 2. Organigrama Ministerio de Educación Pública

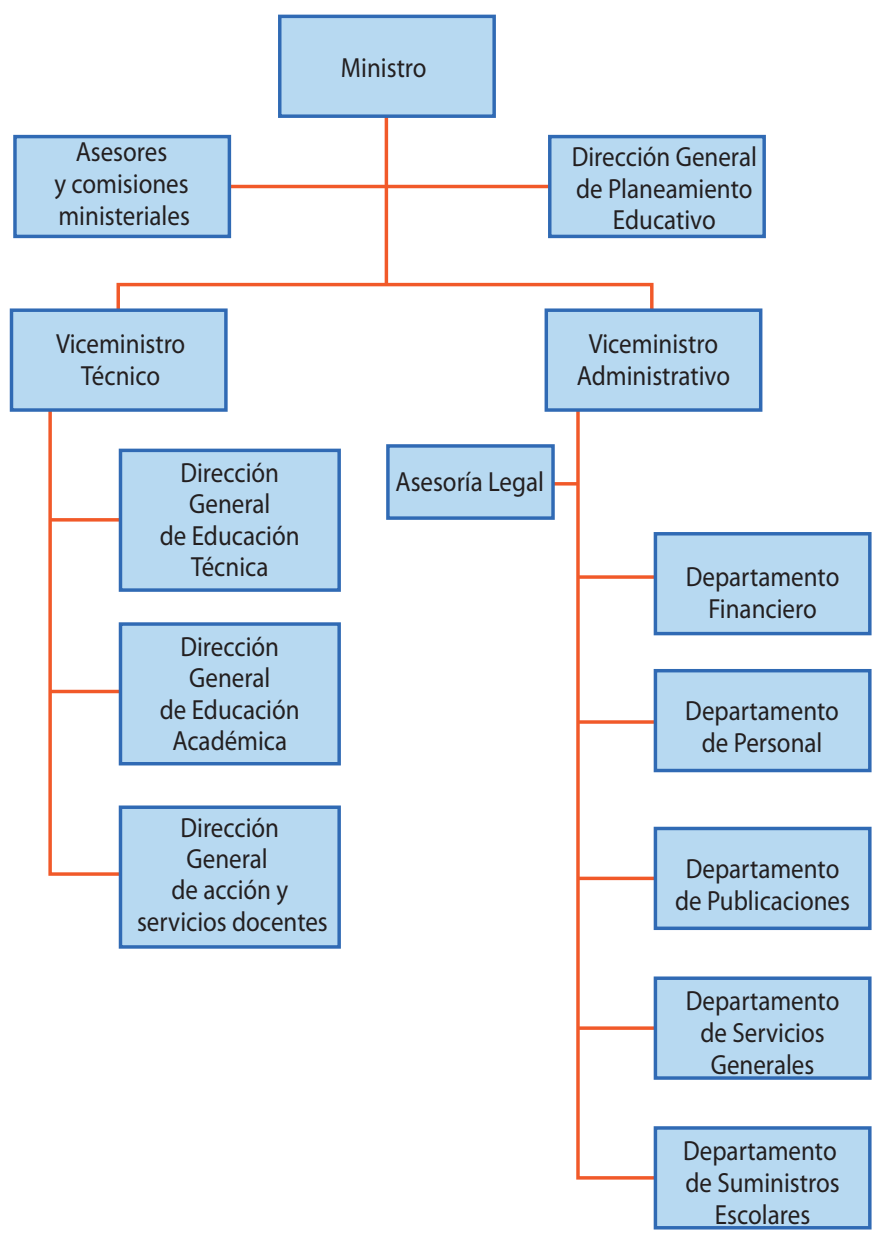

Fuente: Elaboración propia a partir de la propuesta de Gámez (1981).

Los cambios generaron toda una redistribución del resto de unidades y órganos reduciéndolos considerablemente en cantidad. Para el exministro Gámez:

Este organigrama no resiste un estudio crítico sobre el ordenamiento de niveles jerárquicos, nivel político, direcciones generales, departamentos, unidades, secciones, niveles regionales, niveles locales, etc... y se aparta del conveniente y técnico ordenamiento vertical de organismos de planeamiento y asesoramiento. (Gámez, 1981, p. 163) 
En 1977, el Ministro de Educación Pública, Vidal Quirós Berrocal, da cuenta en la memoria institucional que, en cuanto a equipamiento, en 1977 se asignó a las juntas administrativas un total de 590000 colones y se presupuestó la suma de 4410000 colones para la compra de fincas. Además, señala que conscientes de los grandes problemas que acosan a los educadores en el área rural se construyeron 64 casas del maestro rural y se dejaron 27 en proceso de construcción.

Se indica, para 1977, la existencia de 3343 instituciones oficiales de todos los niveles educativos y se cita entre los logros de la administración la planificación de las necesidades de planta física del sistema, conforme con la financiación de "bonos de edificaciones educativas de 1975" por la suma de 60000000 de colones. Se muestran como meta alcanzada los estudios y la realización de gestiones para la construcción de 436 aulas que beneficiaron a 139 instituciones cuya construcción se realizó en coordinación con el Ministerio de Obras Pública y Transportes, además las gestiones ante el Ministerio de Planificación y el Ministerio de Hacienda para la inclusión, en presupuestos nacionales, de partidas para incrementar la infraestructura educativa por un monto de 7591444 colones, por medio de 31 acciones de financiamiento para beneficio de centros educativos.

Entre 1980 y 1982, el país sufre una severa crisis económica y social, la memoria de este periodo, siendo ministro Eugenio Rodríguez Vega, indica:

Hay un dato fundamental que debe tenerse presente por las futuras autoridades: el porcentaje del presupuesto nacional que se dedica a educación ha venido descendiendo en los últimos cinco o seis años. El descenso ha sido sensible, año con año, con motivo de la grave crisis económica que padecen Costa Rica y todas las naciones pobres del mundo: de un $30 \%$ a algo más de un $20 \%$. Un empobrecimiento de esta naturaleza hace más sorprendente lo que hemos podido hacer en estos años de angustias fiscales de verdad asfixiantes. Varios hechos explican que el Ministerio de Educación Pública no se hubiera paralizado: el trabajo devoto de maestros y comunidades, el crédito exterior y el ingreso de generosos recursos extrapresupuestarios. (MEP, 1986, p. 9)

La situación se hacía manifiesta en diferentes áreas de la administración pública, el Ministerio de Educación no es la excepción y en el Alcance $N^{\circ} 11$ a la Gaceta N²8, correspondiente al sábado 8 de mayo de 1982, se publica el decreto 13593-E, llamado Organización Administrativa de las oficinas centrales del Ministerio de Educación Pública, que inicia indicando que dada la complejidad y la diversidad de funciones que el Ministerio de Educación debe prestar, la cantidad de funcionarios que tiene a su servicio y la localización de estos servicios en todo el territorio nacional, se hace necesaria una revisión de su organización y de sus esquemas de trabajo con el fin de adecuarlos a las necesidades actuales de prestación del servicio educativo a fin de que este pueda llegar en forma adecuada a sus destinatarios.

Este decreto señala que la organización se había implantado desde febrero de 1981, siendo Ministra de Educación Pública María Eugenia Dengo Obregón, y que para establecerla se utilizaron criterios que permitieran:

1. Una agrupación por función;

2. unidad de mando sobre las instituciones educativas;

3. mayor coordinación entre las dependencias agrupando éstas por divisiones;

4. delimitación de campos entre divisiones, según su naturaleza; y

5. mayor coordinación regional y de las oficinas centrales del Ministerio. 
Indica también, en su artículo segundo, que para el cumplimiento de su cometido el Ministerio de Educación Pública agrupa sus funciones en las siguientes tres grandes áreas de actividad: el área determinativa, el área de asesoría y el área ejecutiva. Al área de asesoría se le asignó dar el asesoramiento en materia jurídica y de planeamiento que demandara el área determinativa a cargo de la conducción de las políticas educativas, para ser ejecutadas por el área ejecutiva.

Se indica, en la sección III sobre la división de planeamiento y desarrollo educativo responsable de funciones asesoras y ejecutivas, que esta división se organiza en dos direcciones; una dirección general de investigación y una dirección general de planeamiento global que entre sus funciones destaca establecer, conjuntamente con los departamentos de infraestructura y estudios económicos de educación, los criterios para la creación de nuevos centros; específicamente sobre el departamento de planeamiento físico y se señalan como funciones: realizar los estudios de planeamiento físico a fin de determinar las necesidades estructurales del sistema educativo, proponer criterios para la adquisición de terrenos destinados a la educación y mantener una vinculación y coordinación permanente con las instituciones comprometidas en la planificación y en la problemática de la infraestructura educativa.

La memoria de 1982-1986 indica lo siguiente:

Consideremos que la infraestructura educativa comprende terrenos, edificios y equipamiento dedicados al uso de los centros educativos, administrativos, viviendas de docentes y su mantenimiento; Se ejecutan a través de dos secciones especializadas, Estudios Técnicos y Planificación Territorial. La primera se encarga de realizar los análisis previos que se requieren para fundamentar la planificación de la infraestructura de los servicios educativos, la segunda le corresponde analizar las estructuras regional-físicas geográficas y planificar acciones que aseguren el control y registro de los terrenos que se destinan para uso educativo. (MEP, 1986, p. 75)

Presenta también, entre las principales acciones, primero el estudio de solicitudes de construcción para ser incluidas en el programa de construcciones que se elabora en coordinación con la dirección de edificaciones nacionales en el MOPT, segundo el estudio de reconstrucción y construcción de instalaciones educativas afectadas por terremotos en la región sur del territorio nacional en coordinación con el MOPT y tercero la solicitud de recursos a la UNESCO para coadyuvar en la acción, obteniéndose una donación por $\$ 30000$, que se suma a los aportados por la Comisión Nacional de Emergencias, lo cual beneficia a 38 centros educativos. Además, como resultados manifiestan la elaboración de un documento que sintetiza las necesidades reales de construcción en 1985, y se indica que la gestión se encontraba en la Asamblea Legislativa para activar el presupuesto necesario para la construcción de 800 aulas por medio de bonos escolares por 250 millones de colones.

El decreto $\mathrm{N}^{\circ} 13593-\mathrm{E}$ será derogado por uno bastante similar con fecha 10 de octubre de 1985, enumerado decreto $\mathrm{N}^{\circ}$ 16638-MEP (figura 3) y denominado Organización Administrativa de las oficinas centrales del Ministerio de Educación Pública que indica en su artículo uno, que tiene por objeto definir y establecer la organización administrativa de las oficinas centrales del Ministerio de Educación Pública. Entre las principales diferencias con el decreto anterior se identifica que este es más corto y menos especifico en cuanto a funciones. 
Figura 3. Organización MEP, según decreto № 16638 de 1985

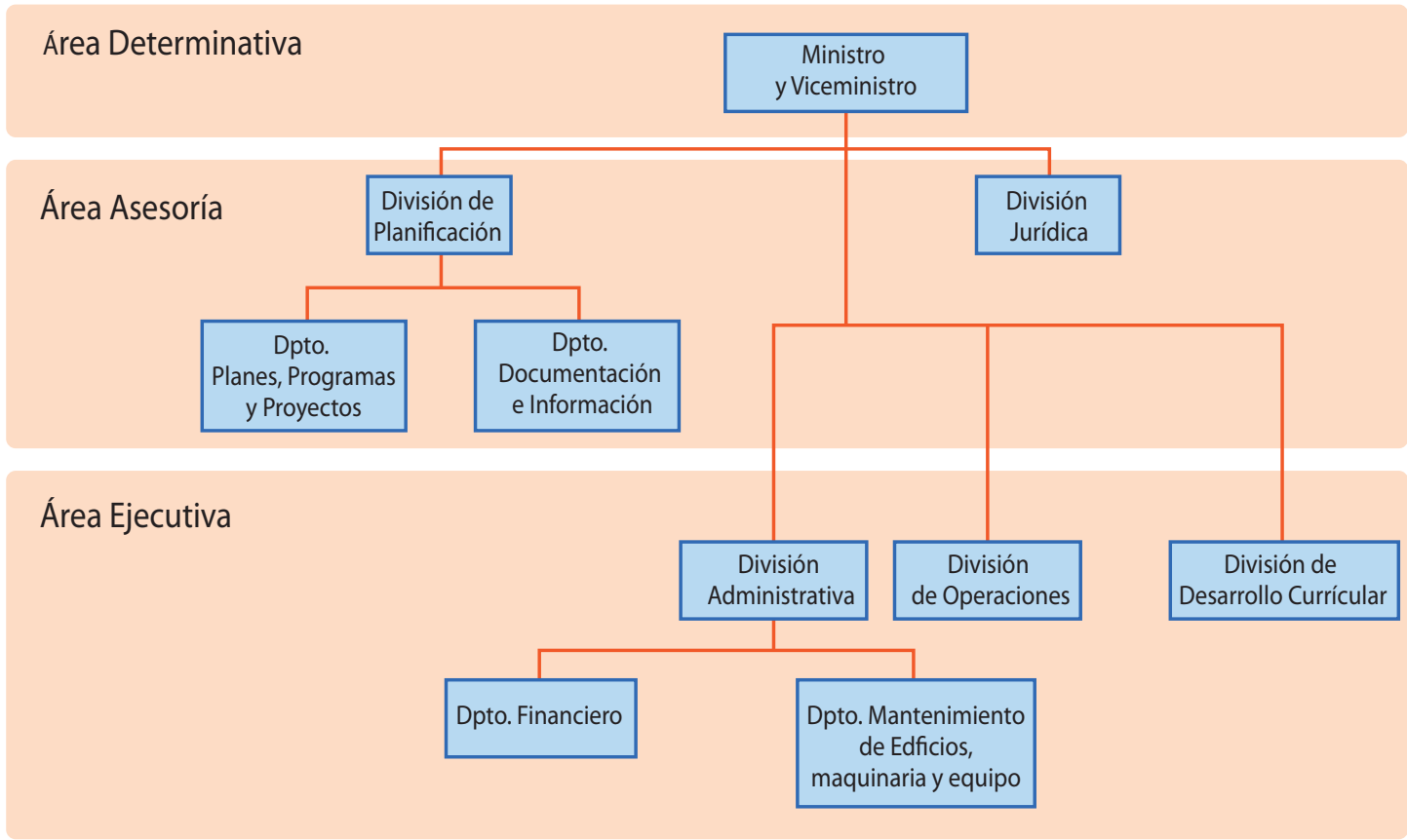

Fuente: Elaboración propia con datos del decreto № 16638 de 1985

Indica este decreto, en su artículo segundo, que para el cumplimiento de su cometido el Ministerio de Educación Pública agrupa sus funciones en las siguientes tres grandes áreas de actividad: primero el área determinativa cuyo ejercicio correspondía al ministro con el auxilio de un viceministro que tenían a cargo la dirección superior, la conducción de políticas educativas, la ejecución de directrices emanadas del Consejo Superior de Educación y el cumplimiento de sus funciones técnico administrativas; segundo el área de asesoría con funciones de orientar y asesorar en funciones de materia jurídica y de planeamiento al área determinativa y ejecutiva para la formulación de políticas y en la definición de estrategias para la toma de decisiones; y tercero el área ejecutiva, a la que le correspondía el cumplimiento de las disposiciones emanadas del área determinativa.

Además se establece que las funciones asesoras y ejecutivas se realizan por medio de cinco divisiones: división jurídica, división administrativa, división de desarrollo curricular, división de operaciones y división de planeamiento y desarrollo educativo; fue a esta última a la que le asignan establecer la coordinación con otros organismos nacionales e internacionales, relacionados con la planeación y el desarrollo educativo.

Así para el cumplimiento de sus funciones, a esta división de planeamiento y desarrollo educativo se le otorga una subdirección, el centro de cómputo, el departamento de planificación administrativa, el departamento de investigación, evaluación y control global, el departamento de planes, programas y proyectos y el departamento de documentación e información.

Las funciones de esta división se distribuyen en dos dependencias, destacando el departamento de planes, programas y proyectos con la función de programar, proyectar y desarrollar estudios sobre necesidades socioeconómicas de la institución, así como sobre planeamiento físico, asuntos internacionales, presupuestos y otros relacionados. Además se le encarga a este departamento programar, proyectar y desarrollar estudios sobre necesidades de recursos humanos, infraestructura, proyectos de desarrollo con financiamiento y asesoramientos externos y otros relacionados. 
Procede también referirnos a otra división, la denominada división administrativa encargada de ejecutar acciones para el desarrollo del sistema educativo en cuanto a recursos humanos, físicos y financieros; ejecutar planes y programas de inversión según políticas emanadas del nivel superior; establecer normas y procedimientos para una adecuada administración de los recursos disponibles; aplicar sistemas de organización y trabajo de probada eficiencia; propiciar planes, programas y actividades de formación en servicio para los funcionarios con responsabilidad administrativa; y ejecutar la acción administrativa en un ambiente de óptimas relaciones humanas y de eficiente servicio al público.

Esta referencia se hace por cuanto la división contaba, para el cumplimiento de su cometido, con varios departamentos entre los que vale mencionar dos: primero al departamento financiero encargado de administrar el presupuesto y los fondos que ingresaran al Ministerio de Educación Pública, tanto en presupuesto ordinario y sus modificaciones como otras leyes que asignaran recursos para la educación; y segundo con los organismos internacionales y de otros gobiernos.

Para octubre de 1986, esta forma de organización es criticada por la misión enviada al país por la Organización de las Naciones Unidas para la Educación, la Ciencia y la Cultura (UNESCO) a solicitud del Ministro de entonces, Francisco Antonio Pacheco.

Esta estructura relativamente sencilla ha sido diseñada con el propósito de responder, en forma ágil a los requerimientos complejos y diversificados planteados por un servicio educativo en proceso de desconcentración y regionalización. Ella parece convenir, en líneas generales, para un ejercicio eficiente de las funciones que incumben al MEP, la misión estima conveniente, sin embargo, que el proceso de organización iniciado con la promulgación del decreto $\mathrm{N}^{\circ} 16638$, sea reforzado con vistas a aliviar problemas de centralismo, burocratización e ineficiencia, ya que subsisten algunas dificultades de ajuste a las que convendría encontrar rápida solución. (UNESCO, 1986, p. 3)

Esta organización vincula inmediatamente al despacho del Ministro con un número relativamente elevado de órganos, lo que seguramente se traduce en una congestión de asuntos diversos sobre el despacho del Ministro. Además indica el informe que la división de planeamiento y desarrollo educativo cuenta con 110 empleados distribuidos en los diferentes departamentos. Otro problema señalado en el informe es la carencia a nivel de la programación, lo que conducirá a situaciones de improvisación y que el ejercicio de las funciones se vea entorpecido por la carencia de un plan anual de actividades y subactividades que debe ser ejecutado a cada nivel de cada una de las distintas divisiones, departamentos y secciones que integran el nivel central.

Se advierte también en el informe de la UNESCO lo siguiente:

Como resultado de las informaciones obtenidas con ocasión de las entrevistas efectuadas y de la consulta a entes autorizados y a los diversos documentos y estudios cabe señalar que la planificación educativa que ha atravesado Costa Rica en la década pasada por un periodo de gran eficiencia y aceptación se ha atrincherado en los últimos años en el desarrollo de acciones puntuales, no programadas, en apoyo a expedientes y proyectos no forzosamente vinculados a un proceso de planificación integral. (UNESCO, 1986, p. 3)

Soluciones puntuales son desarrolladas para solventar los vacíos del reglamento, sin necesidad de crear un nuevo decreto completo de reorganización administrativa. Mención especial merece el decreto $\mathrm{N}^{\circ}$ 18933-MEP del 27 de marzo de 1989, que crea el Centro Nacional de Infraestructura Física Educativa (CENIFE), trasladándosele las competencias de la División de planeamiento y desarrollo educativo y las del Departamento de Mantenimiento de Edificios, Equipo y Mobiliario de la División Administrativa. La memoria del periodo 1986-1990 del ministro Pacheco, indica que al asumir la gestión ministerial se 
señaló como uno de los principales problemas el faltante, que existía en esa fecha, superior a 4000 aulas y más de 30000 pupitres.

También señala el documento que una de las decisiones que más ayudó al éxito en la construcción y mantenimiento de la infraestructura escolar fue la creación del CENIFE con la función de promover y desarrollar, desde una perspectiva integral, modelos infraestructurales en las áreas de construcción, mantenimiento, renovación y equipamiento de sistema educativo dependiente del MEP, lográndose mejorar las condiciones ambientales en procura de garantizar la eficiencia de la educación en el país.

Para atender el problema de falta de infraestructura escolar esta administración recurre a varios programas, un programa de renovación escolar que mediante un financiamiento de 120 millones de colones, procedente de la A.I.D. (Agencia de los Estados Unidos para el Desarrollo Internacional), beneficia a 553 escuelas rurales; un programa denominado Ley de bonos, para completar 800 aulas, indicándose que a la fecha se habían construido 600 aulas, quedando pendientes 200 para el año de 1990 y señalándose la ampliación de esta ley para construir 400 aulas más; además la comisión MEP/MOPT permite que el MOPT contrate con empresas constructoras la edificación de obras que beneficiaría a 188 centros educativos.

Como logros puntuales, la memoria citada manifiesta la creación del Departamento de Proveeduría definiéndosele su estructura organizativa, contando para ello con personal altamente calificado y se le da mérito a la División Jurídica en lo referente a inscripción de propiedades a nombre del Ministerio, recibiendo incluso felicitación verbal de la notaría del Estado.

El 9 de diciembre de 1991 el gobierno de Costa Rica toma la decisión de suscribir un contrato de préstamo con el Banco Internacional de Reconstrucción y Fomento (Banco Mundial), por un monto de veintitrés millones de dólares estadounidenses, seguidamente el 19 de diciembre de 1991, el gobierno contrata un empréstito con el Banco Interamericano de Desarrollo (B1D) por un monto de veintiocho millones de dólares estadounidenses, ambos para la financiación del proyecto denominado Programa para el Mejoramiento de la Calidad de la Educación Básica (PROMECE).

La descripción del programa incluye, entre otros elementos, algunos propios del área de infraestructura: rehabilitar aproximadamente 2300 aulas; construir y reemplazar aproximadamente 240 aulas para el nivel de educación primaria, incluyendo el suministro y la utilización de mobiliario, equipo e instalaciones sanitarias; construir aproximadamente 110 casas para maestros de zonas marginales y construir y equipar cuatro nuevos direcciones regionales, localizadas en los cantones de Liberia, Puntarenas, San Carlos y Pérez Zeledón y además de remodelar dos de los seis sedes regionales existentes, localizadas en San José y Limón.

Para 1992, los empréstitos son aprobados por la Asamblea Legislativa de Costa Rica; En el documento se observa un capítulo IV de normas relativas a desembolsos, con el requerimiento del Banco Interamericano de Desarrollo para que el gobierno de Costa Rica presente evidencia de la creación de una unidad coordinadora del proyecto "UCP" cuyo director haya sido designado y además que el personal integrante también haya sido seleccionado y se encuentre trabajando con el reglamento de su organización y operación. Como resultado, el Poder Ejecutivo, por medio del decreto № 21903 del 02 de febrero de 1993, emite el Reglamento de Creación y Funcionamiento de la Unidad Coordinadora del Programa de Mejoramiento de la Calidad de la Educación General Básica, como órgano adscrito al despacho del Ministro de Educación Pública.

Entre las funciones se determinan las siguientes: coordinar las actividades del programa a cargo de unidades ejecutoras del Ministerio de Educación Pública; servir de enlace para efectos de coordinación y decisión entre el MEP, el BID y el Banco Mundial; dirigir, coordinar, supervisar y responder por el desarrollo técnico, financiero y administrativo del programa; preparar para su respectiva aprobación los planes operativos en forma detallada y los presupuestos del programa, así como supervisar la adecuada 
y oportuna ejecución de los mismos; evaluar periódicamente el programa y recomendar las modificaciones que se estimaran pertinentes.

Para el funcionamiento de la unidad coordinadora del proyecto, se establece que cuente con una dirección, una sección financiero-administrativa, una sección técnica, una comisión de adjudicaciones y una comisión asesora integrada básicamente por los anteriores, más los jerarcas de las unidades ejecutoras; estas últimas definidas como órganos de apoyo ajenos a la estructura orgánica de la unidad coordinadora del proyecto, con responsabilidades en los siguientes campos: infraestructura física, adecuación curricular, evaluación, informática educativa y de gestión, recursos para el aprendizaje, desarrollo profesional y desarrollo institucional.

Prácticamente un mes después de constituido PROMECE, se establece un nuevo reglamento de organización, publicado en La Gaceta № 48 del 10 de marzo de 1993, el decreto № 21896-MEP denominado La Organización Administrativa de las Oficinas Centrales del Ministerio de Educación Pública, el decreto conserva la estructura básica por áreas y no incluye los decretos puntuales, si no que los mantiene vigentes, de forma que no afectara al CENIFE.

EI CENIFE es organizado desde su decreto de creación con una dirección ejecutiva a cargo de un director ejecutivo y de un subdirector administrativo, con tres departamentos subordinados: departamento de equipamiento, departamento de normas y diseños y el departamento de planificación y desarrollo; sin embargo por medio de decreto $\mathrm{N}^{\circ} 23140$ del 27 de abril de 1994 se reforma el decreto original que crea el CENIFE, explicando que debido a la experiencia adquirida hasta la fecha, resultaba imperioso integrar las tareas de planeamiento físico y diseño arquitectónico de los centros educativos, con las del control de la ejecución, por lo que aumentan los departamentos y se reagrupan con la subdirección ejecutiva, al departamento de arquitectura escolar, al departamento de equipo e instalaciones, a la unidad financiera, a la unidad de informática y a la unidad de apoyo secretarial.

Para el mes de julio de ese año, surge el decreto № 23489: Reforma Organización Administrativa Oficinas Ministerio Educación, que aclara que el CENIFE está bajo la responsabilidad del Viceministerio de Educación. De acuerdo con la memoria institucional del año 2001, siendo ministro Guillermo Vargas Salazar, al 31 de diciembre de ese año se realiza una inversión que ascendió a 3500 millones de colones, el financiamiento de 1372 aulas y, por otra parte, se indica la transferencia de recursos del presupuesto nacional a las juntas de educación y administrativas con la finalidad de que ellas asuman directamente la solución de sus problemas de infraestructura escolar; se agrega también que con recursos externos del Programa de Mejoramiento de la Calidad de la Educación (PROMECE) fue posible construir 54 aulas con sus respectivas baterías de servicio.

El 14 de mayo de 2002 el decreto $N^{\circ} 30323$ crea el Instituto de Investigación y Desarrollo de la Infraestructura Educativa Costarricense (IDIECO), como órgano de desconcentración máxima del Ministerio de Educación Pública basado en que el crecimiento de la población en edad escolar, así como la diversificación y enriquecimiento curriculares demandaban en los últimos diez años un significativo aumento de la infraestructura escolar que no fue debida y oportunamente satisfecho por lo que existía un déficit histórico de más de 5200 aulas para el año 1998.

La creación de IDIECO es derogada para el 09 de octubre de 2002 y se pone en vigencia y reforma el decreto $N^{\circ} 18933$ de creación del CENIFE. Según el decreto №30753 de la derogación, se señala que la presente administración estima que no es el momento idóneo para ejecutar reestructuraciones administrativas que no están suficientemente respaldadas desde el punto de vista técnico y administrativo y adolecen de una debida y oportuna previsión presupuestaria, además se indica que las reformas introducidas afectan notoriamente no sólo este mismo proceso de transición, sino la eficiencia de las dependencias involucradas y la seguridad jurídica y estabilidad emocional de los clientes, usuarios y funcionarios relacionados con los servicios y funciones atinentes a ellas. 
Otras reformas se llevan a cabo en la organización del Ministerio de Educación Pública, mediante decretos: $\mathrm{N}^{\circ} 23490$ del 29 de julio de 1994, N 30303 del 17 de abril de 2002, $\mathrm{N}^{\circ} 30376$ de agosto de 2002; sin afectar al CENIFE, hasta que el 5 de noviembre de 2007 se publica el decreto $N^{\circ} 34075-M E P$ denominado: Organización administrativa de las oficinas centrales del Ministerio de Educación Pública, que deroga la legislación del CENIFE y establece la Dirección de Infraestructura y Equipamiento Educativo (DIEE).

La constitución de la DIEE como asesoría técnica al Consejo Superior de Educación (CSE) para el cumplimiento de sus competencias constitucionales y legales relacionadas con la infraestructura educativa, logra definir a esta dirección como el órgano encargado de planificar, desarrollar, coordinar, dirigir, dar seguimiento y evaluar planes, programas y proyectos tendientes al mejoramiento y ampliación de la infraestructura física educativa y su equipamiento, como medio para facilitar el acceso, la calidad y la equidad de la educación pública costarricense. Además, entre otras funciones se le encarga asesorar a las juntas de educación y juntas administrativas en la planificación e implementación de los proyectos de infraestructura educativa y equipamiento, financiados con recursos transferidos por el MEP y otras fuentes de financiamiento.

Para el cumplimiento de las funciones se le otorgan a la DIEE cuatro departamentos: el departamento de investigación y desarrollo, el departamento de proyectos, el departamento de contrataciones y el departamento de ejecución y control. Esta dirección y sus departamentos sobrevivirían al decreto $\mathrm{N}^{\circ} 36451$, publicado en el diario oficial La Gaceta del miércoles 9 de marzo del 2011 y al decreto №38170, publicado en el diario oficial La Gaceta del jueves 13 de febrero del 2014, con el cual se fortalece la DIEE con una nueva departamentalización integrada por el Departamento de desarrollo de infraestructura educativa, el Departamento de investigación, el Departamento de gestión de proyectos específicos, el Departamento de contrataciones y el Departamento de ejecución y control (ver organigrama mostrado en la figura 4).

Figura 4. Organización MEP, según decreto № 38170 de 2014

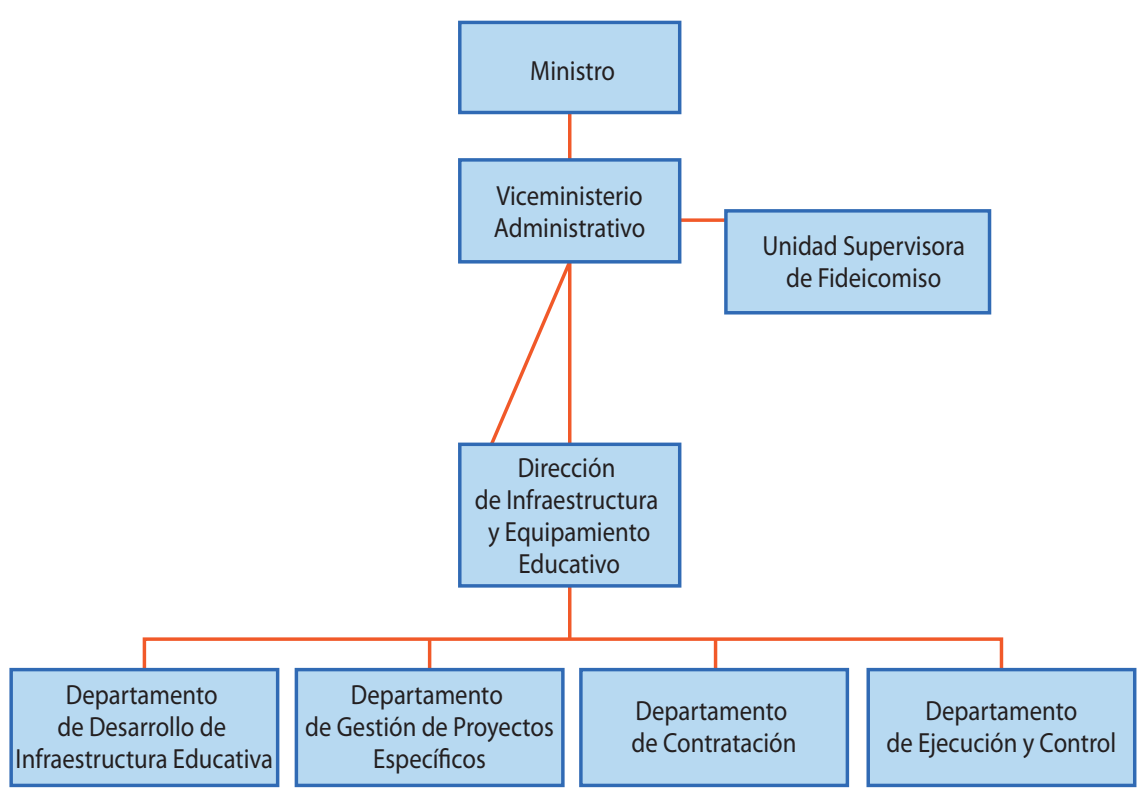

Fuente: Elaboración propia con datos del decreto N33170 de 2014. 
El decreto $N^{\circ} 38170$ incorpora además la Unidad Supervisora del Fideicomiso (USP) de la Ley $N^{\circ} 9124$, una contraparte técnica y administrativa del Ministerio de Educación Pública, dependiente directamente del Viceministerio Administrativo, con funciones de supervisión y seguimiento, de un nuevo mecanismo de financiamiento para desarrollar obras educativas, mediante el fideicomiso aprobado por la Asamblea Legislativa de Costa Rica el 21 de marzo de 2013, que permite por primera vez que una entidad del gobierno central pueda recurrir a ese tipo de instrumentos financieros para acelerar significativamente la capacidad de contar con infraestructura educativa.

El proyecto del fideicomiso educativo del Ministerio de Educación Pública incluye el diseño, la construcción, el equipamiento y el mantenimiento de 79 centros educativos completos y 24 canchas multiuso, proyectando beneficiar a alrededor de 40000 estudiantes en todo el territorio nacional. En junio de 2013 el MEP contrata los servicios del Banco Nacional de Costa Rica (BNCR) como Banco Fiduciario y este a su vez en enero de 2014, suscribe un contrato de préstamo por 167,5 millones de dólares con el Banco Interamericano de Desarrollo (BID), para el financiamiento del proyecto.

De acuerdo con la memoria institucional del MEP 2010-2014, se señala que:

En el 2006, la inversión anual en infraestructura no llegaba a los \7 mil millones; no existía casi personal profesional asignado a esta tarea; casi no había vehículos disponibles y tampoco se tenían otras opciones de financiamiento. En los últimos cinco años (2010 a 2014) la inversión presupuestada fue, en promedio, de $\$ 38$ mil millones por año, un aumento notable al que se suman ahora los recursos del fideicomiso educativo. (MEP, 2014, p. 351)

La memoria institucional del MEP 2014-2018 expone que para el cierre del informe, utilizando la figura de fideicomiso se logra concluir tres proyectos, adicionalmente, quince proyectos se encontraban en ejecución y treinta y nueve proyectos de infraestructura educativa se encontraban en realización de planos constructivos.

\section{RESULTADOS}

La comprensión de la evolución del MEP, en su búsqueda por atender la necesidad de edificios escolares, se evidencia en lo extenso que ha sido el proceso para el organismo gubernamental, al abarcar desde 1965 al año actual 2020; 55 años sin alcanzar una solución definitiva a la problemática; las causas son evidenciadas en la bibliografía analizada: la insuficiente cantidad de recursos y la lentitud de los procesos administrativos que incluyen entre otros los procesos de planificación, presupuestación, organización, control y contratación de obra pública; sin mencionar las circunstancias propias que afectan los procesos constructivos.

La tabla 1 presenta las fases en que se puede aglutinar la evolución de la organización ministerial para enfrentar el problema 
TABLA 1

Fases evolutivas de la organización del MEP para atender la necesidad de infraestructura educativa

Fases Papel institucional Caracterización

Fase 1. Pre-Ministerio

Personas o entidades públicas o privadas construyen edificaciones escolares sin necesidad de autorizaciones.

Fase 2. Ministerio - Juntas de educación y administrativas. (A partir de 1965 con Ley № 3481 denominada: "Ley Orgánica del Ministerio de Educación Pública")
MOPT- MEP y juntas de educación y administrativas desarrollan las obras de infraestructura escolar.
Niveles variables o desconocidos de calidad de las obras, con nulos o pocos detalles de procesos licitatorios.
Fase 3. Especialización del MEP en Infraestructura educativa. (A partir de 1989 con el decreto $N^{\circ} 18933-M E P$ que crea el CENIFE)
Se evidencian conflictos político -administrativos. Planificación de atención de las necesidades de planta física educativa.

Dispersión de funciones en diferentes departamentos.

Es evidente la falta de recursos financieros.
Fase 4. Administración de proyectos financiados con créditos externos. A partir del decreto $N^{\circ} 21903$ del 02 defebrero de 1993 que emite el Reglamento de Creación y Funcionamiento de la Unidad Coordinadora de PROMECE.
Se establecen entidades internas al MEP focalizadas en atender el déficit de infraestructura.
Mejora en la identificación y solución de necesidades para brindar el servicio educativo.

Organización voluble y expuesta a definiciones políticas.
Se constituyen unidades ejecutoras de proyectos; cuya labor es fiscalizada por el MEP.
La ejecución de los recursos y desarrollo de proyectos es supervisada por bancos internacionales; Ministerio de Hacienda y juntas de educación y administrativas.

Fuente: Elaboración propia (2020).

Las fases, aunque se puedan traslapar, muestran la variabilidad de la organización fundamentada en legislación que le da sustento jurídico a la misma para transformarse. De acuerdo con Idalberto Chiavenato, las organizaciones son extremadamente heterogéneas y diversas, cuyo tamaño, características, estructuras y objetivos son diferentes (2006, p. 2).

Las soluciones observadas en el estudio evolucionaron a la búsqueda de recursos financieros provenientes de organismos internacionales como el Banco Mundial y el BID; y la constitución legal de órganos de gestión de proyectos alternativos focalizados en el desarrollo de la infraestructura escolar, que procuran mantenerse al margen de decisiones políticas como lo pueden ser la priorización de proyectos o la asignación de recursos presupuestarios definidos.

En el contexto, el valor de las juntas de educación y juntas administrativas se encuentra en ser unidades de gestión concentradas en dar solución a su problemática local; sin embargo, las circunstancias que internamente puedan afectar a estos colectivos sociales, como podrían ser la poco o nula experiencia en el desarrollo de proyectos de infraestructura pública o en la administración de recursos estatales, constituyen retrocesos o demoras indeterminadas en plazo, para resolver la necesidad de las obras.

El surgimiento del Fideicomiso de Infraestructura Educativa es correspondiente con la identificación de un instrumento para la búsqueda de recursos financieros y es congruente en seleccionar una unidad responsable de la ejecución del proyecto con conocimiento y criterio técnico para gestionar proyectos durante plazos mayores a los periodos presidenciales de cuatro años. 


\section{CONCLUSIONES}

El déficit en materia de infraestructura educativa se ha posicionado en las últimas décadas como el principal problema estructural del área administrativa del Ministerio de Educación Pública, cuya atención seguirá requiriendo del apoyo decidido de las sucesivas autoridades. Identificar una organización que logre satisfacer este requerimiento continuará siendo, en el corto y mediano plazo, un desafío que se debe atender si se espera que la reducción del déficit de infraestructura escolar y la construcción de espacios recreativos y deportivos tengan como resultado un mejor clima escolar y mayor motivación de docentes y estudiantes.

De la revisión documental hecha anteriormente, se desprende que históricamente el Ministerio de Educación Pública, como ente rector de la educación costarricense ha creado y aprobado la normativa propia de la época, para proveer a las comunidades de la infraestructura física educativa adecuada a las necesidades de las personas estudiantes.

En el campo de la articulación interinstitucional llama la atención el vínculo inicialmente establecido entre el MEP y el MOPT con el denominado Departamento de Ayudas Comunales, como una dependencia más abocada también al apoyo en gestión de infraestructura educativa.

De igual manera, en el apartado de financiamiento externo, sobresale la creación de PROMECE, como fruto del préstamo con el Banco Mundial y el Banco Interamericano de Desarrollo y cuyo fin específico era también el desarrollo de construcción de obra educativa.

De los esfuerzos propiamente institucionales por parte del MEP, destacan principalmente la creación del CENIFE y la Dirección de Infraestructura y Equipamiento Educativo, como última legislación específica en materia de infraestructura y equipamiento.

Pero quizás una de las legislaciones más ambiciosa se logran visualizar en la Ley № 9124 que constituye el fideicomiso en el que, mediante préstamo con el Banco Interamericano de Desarrollo se autoriza, por parte de la Asamblea Legislativa, al Ministerio de Educación Pública a constituir la figura del fideicomiso, adjudicando posteriormente a un banco fiduciario con unidad ejecutora, con la misión de concretar, en plazos de ley importantes, obras educativas en el territorio nacional. Este se convierte en un esfuerzo importante por buscar más y mejores alternativas de financiamiento y ejecución de obra, que vislumbra en las alianzas público-privadas una posibilidad real de concretar obras.

La innovadora figura del fideicomiso de la Ley $N^{\circ} 9124$, representa la conciencia de que el déficit de infraestructura educativa es un problema de tales dimensiones que exceden a la capacidad limitada de una sola organización como el MEP y que, por lo tanto, se requiere de un mayor nivel de involucramiento de actores públicos y privados, si es que el país se plantea como firme objetivo el poder brindar, tanto a educadores como a educandos, las condiciones mínimas de infraestructura (así como mobiliario, material didáctico, etc.) para garantizar la tutela del derecho fundamental a la educación pública, en los términos establecidos en la Constitución Política, lo que implica la prestación de un servicio público gratuito, de calidad y costeado por la Nación. 


\section{REFERENCIAS}

Arce, C. (2004). Constitución Política de la República de Costa Rica. Debidamente actualizada con las Reformas adicionales y resoluciones de la sala constitucional al primero de mayo del 2004. San José, C.R., 2004.

Arce, C. (2012). Derecho Educativo. Costa Rica: Editorial Universidad Estatal a Distancia.

Chiavenato, I. (2006). Introducción a la teoría general de la administración (7ª. edición), México, McGraw-Hill, 2006.

Decreto №3333-E. Diario Oficial La Gaceta, San José, Costa Rica, 21 de noviembre de 1973.

Decreto Nº 13593-E. Diario Oficial La Gaceta, San José, Costa Rica, 08 de mayo de 1982.

Decreto Nº 18933-MEP. Diario Oficial La Gaceta, San José, Costa Rica, 27 de abril de 1987.

Decreto № 21896-MEP. Diario Oficial La Gaceta, San José, Costa Rica, 10 de marzo de 1993.

Decreto N²1903-MEP. Diario Oficial La Gaceta, San José, Costa Rica, 11 de marzo de 1993.

Decreto N²7917-MOPT. Diario Oficial La Gaceta, San José, Costa Rica, 10 de junio de 1999.

Decreto N 30323-MEP. Diario Oficial La Gaceta. San José, Costa Rica, 14 de mayo de 2002.

Decreto N 34075-MEP. Diario Oficial La Gaceta. San José, Costa Rica, 05 de noviembre de 2007.

Decreto N 36451-MEP. Diario Oficial La Gaceta. San José, Costa Rica, 09 de marzo de 2011.

Decreto № 38170-MEP. Diario Oficial La Gaceta. San José, Costa Rica, 13 de febrero de 2014.

De la Cruz, V. Fischel, A. Quesada, J. Dengo, M. González, Y. Araya, C. Fournier, E. Retana, C. \& Rojas, Y. (2003). Historia de la Educación Costarricense. Costa Rica: Editorial Universidad Estatal a Distancia.

Fulmer, R. (1983). Serie de compendios científicos, Administración y Organización. México: Compañía Editorial Continental, S.A de C.V.

Gámez, Uladislao. (1981). Política y Estructura del Sistema Educativo. Costa Rica: Editorial Universidad Estatal a Distancia.

Hernández, Rubén. (2008). Constitución Política de la República de Costa Rica Actualizada, Comentada, anotada y con citas de jurisprudencia. Costa Rica: Editorial Juricentro.

Jinesta, Ernesto. (2011). Tutela y descentralización administrativas. X Foro Iberoamericano de Derecho Administrativo, Retos de la Organización administrativa contemporánea, El Salvador.

Ley N 1362, Creación del Consejo Superior de Educación Pública. San José, Costa Rica. 08 de octubre de 1951.

Ley N 2160. San José, Costa Rica. 02 de octubre de 1957.

Ley № 8558. Diario Oficial La Gaceta. San José, Costa Rica, 30 de noviembre de 2006.

Ley Nํ 9124. Diario Oficial La Gaceta. San José, Costa Rica, 22 de marzo de 2013.

Ley N³481, Ley Orgánica del Ministerio de Educación Pública del año 1965.

Lépiz, Carlos. (2003). Administración de la Educación, La administración y planificación como procesos. Costa Rica: Editorial Universidad Estatal a Distancia.

Ministerio de Educación Pública. (1977).Memoria 1977. San José: MEP.

Ministerio de Educación Pública. (1986).Memoria 1985 Periodo 1982-1986. San José: MEP.

Ministerio de Educación Pública. (1990).Memoria final Periodo 1986-1990. San José: MEP. 
Ministerio de Educación Pública. (2001).Memoria institucional Administración 1998-2002. San José: MEP.

Ministerio de Educación Pública. (2010).Memoria institucional 2006-2010 Educando en tiempos del cambio. San José: MEP.

Ministerio de Educación Pública. (2014).Memoria institucional 2006-2014 La educación subversiva: atreverse a construir el país que queremos. San José: MEP.

Ministerio de Educación Pública. (2018).Memoria institucional Administración 2014-2018 Educar para una nueva ciudadanía: una poderosa transformación del sistema educativo. San José: MEP.

Tantaleán, R. (2016) Tipología de las investigaciones jurídicas ISSN: 2224-413. Depósito legal: 20055822. Cajamarca. Perú. Editorial Derecho y cambio social.

UNESCO. (1986). Examen de la problemática educativa y propuesta sobre estrategias de planificación educativa y planta física en Costa Rica. San José: OREALC-UNESCO.

White, Jay (1999). Taking Language Seriously. The Narrative Foundations of Public Administration Research, Washington D.C: Georgetown University Press.

Para citar este documento:

Víquez-Duarte, M. y Orozco-Delgado, V. (2020). La administración de proyectos de infraestructura en el Ministerio de Educación Pública de Costa Rica. Revista Innovaciones Educativas. 22(32), 213-231. DOI: 10.22458/ ie.v22i32.2819 
